\title{
Why should we care about upper airway inflammation in obstructive sleep apnoea?
}

\author{
Malcolm Kohler ${ }^{1,2}$
}

Affiliations: ${ }^{1}$ Dept of Pulmonology, University Hospital of Zurich, Zurich, Switzerland. ${ }^{2}$ Interdisciplinary Center for Sleep Research, University of Zurich, Zurich, Switzerland.

Correspondence: Malcolm Kohler, Dept of Pulmonology, University Hospital of Zurich, Raemistrasse 100, 8091 Zurich, Switzerland. E-mail: Malcolm.Kohlerdusz.ch

@ERSpublications

Treatment of upper airway inflammation may be a novel therapeutic approach in obstructive sleep apnoea http://ow.ly/jeal302QSqc

There is a number of reasons why the article by VINCENTE et al. [1] published in this issue of the European Respiratory Journal deserves a second thought. It is one of the very few studies that addresses the hypothesis that there may be some "spill-over" effect of inflammation from the upper airway into the blood circulation in patients with obstructive sleep apnoea (OSA) [1-3]. According to this hypothesis, which has also been proposed as a mechanism to explain raised blood inflammatory marker levels in other diseases, such as chronic obstructive pulmonary disease [4], local inflammation results in an increased production of inflammatory cytokines and proteins that reach the plasma. These enhance systemic inflammation and thereby contribute to pathophysiological effects of chronic inflammation, such as disturbed lipid and glucose metabolism, and ultimately, development of atherosclerosis. However, recently the spill-over hypothesis has been discussed controversially [4].

VINCENTE et al. [1] present an elegant approach to evaluate a possible association between local upper airway inflammation and systemic disease; the authors collected pharyngeal lavage (PHAL) and plasma at the same time, and determined levels of several pro-inflammatory markers, such as interleukin (IL)-6, IL-8, tumour necrosis factor- $\alpha$ and C-reactive protein, and leukocytes in OSA patients $(n=89)$, snorers $(n=28)$ and healthy control subjects $(n=26)$. Participants were re-assessed 12 months after their initial visit. Interestingly, VINCENTE et al. [1] found higher IL-6, IL-8 and CD4 ${ }^{+}$T-cells in the PHAL of severe OSA patients (apnoea-hypopnoea index $(\mathrm{AHI}) \geqslant 30$ per $\mathrm{h}$ ) compared to the snoring and healthy control groups. In addition, there was an association of PHAL levels of IL-6, IL-8 and CD4 ${ }^{+}$T-cells with AHI, and treatment with continuous positive airway pressure (CPAP) for 12 months resulted in decreased PHAL levels of these inflammatory markers. In contrast, none of the inflammatory cytokines or any subtype of lymphocyte levels in plasma differed between patients with severe OSA and control groups. There was also no association between plasma and PHAL inflammatory markers, and last but not least, CPAP treatment had no effect on plasma inflammatory markers.

What do these findings tell us? The authors correctly state in the discussion of the manuscript that to definitely reject the spill-over hypothesis, one would possibly need a specific inflammatory marker produced in the upper airway that could then be measured in blood. The inflammatory cytokines used in the study by VINCENTE et al. [1] are those that are proposed in the literature to stem from OSA-induced intermittent hypoxia and oxidative stress, and have been associated with detrimental effects on endothelial structure and function [5]. As there was no association between PHAL and plasma levels of these inflammatory markers, and CPAP only had an effect on local inflammation, one must conclude that upper airway inflammation in OSA patients is not contributing to the possibly clinically more relevant systemic inflammation.

Received: June 232016 | Accepted: July 092016

Support statement: This work was supported by Swiss National Science Foundation grant 32003B_162534/1 and the University of Zurich Clinical Research Priority Programme Sleep and Health. Funding information for this article has been deposited with the Open Funder Registry.

Conflict of interest: Disclosures can be found alongside this article at erj.ersjournals.com

Copyright OERS 2016 
This study also adds some additional evidence to the debate of whether or not OSA is associated with systemic inflammation [6,7]. Although it has all the limitations of a case-control study and was not primarily designed to assess the effects of CPAP therapy on systemic inflammatory markers, the authors did not find any association between OSA severity and plasma inflammatory markers, and also found no effect of CPAP on these markers. The study thus corroborates the findings of multiple randomised controlled trials showing no effect of CPAP on systemic inflammatory markers [8-11].

Perhaps the most important point is that VINCENTE et al. [1] draw our attention to a potential new treatment approach for OSA. There is some preliminary work suggesting that local and systemic inflammation may impair respiratory muscle contractility and muscle control function [12-14]; thus, local upper airway mucosal inflammation may promote upper airway muscle dysfunction and contribute to airway collapse in OSA patients [15]. Moreover, it has been suggested that ongoing inflammation and repair may lead to remodelling of the upper airway, and increased deposition of connective tissue in the mucosa and in some of the upper airway muscles $[16,17]$. Of course, the swelling of the pharyngeal mucosa and proliferation of connective tissue narrow the intraluminal pharyngeal cross-sectional area and, thus, contribute to upper airway obstruction during sleep. Therefore, if there was a medication reducing pharyngeal mucosa inflammation and swelling effectively, this could provide a novel treatment option to be explored in OSA.

\section{References}

1 Vincente E, Marin JM, Carrizo SJ, et al. Upper airway inflammation and systemic inflammation in obstructive sleep apnoea. Eur Respir J 2016; 48: 1108-1117.

2 Hauber HP, Rüller S, Müller E, et al. Pharyngeal lavage lymphocytosis in patients with obstructive sleep apnea: a preliminary observation. PLoS One 2011; 6: e16277.

3 Kimoff RJ, Hamid Q, Divangahi $\mathrm{M}$, et al. Increased upper airway cytokines and oxidative stress in severe obstructive sleep apnoea. Eur Respir J 2011; 38: 89-97.

4 Sinden NJ, Stockley RA. Systemic inflammation and comorbidity in COPD: a result of overspill of inflammatory mediators from the lungs? Review of the evidence. Thorax 2010; 65: 930-936.

5 Kohler M, Stradling JR. Mechanisms of vascular damage in obstructive sleep apnea. Nat Rev Cardiol 2010; 7: 677-685.

6 Kohler M, Stradling JR. CrossTalk proposal: most of the cardiovascular consequences of OSA are due to increased sympathetic activity. J Physiol 2012; 590: 2813-2815.

$7 \quad$ Lavie L, Lavie P. Rebuttal from Lena Lavie and Peretz Lavie. J Physiol 2012; 590: 2823.

8 Kohler M, Ayers L, Pepperell JC, et al. Effects of continuous positive airway pressure on systemic inflammation in patients with moderate to severe obstructive sleep apnoea: a randomised controlled trial. Thorax 2009; 64: 67-73.

9 Kohler M, Stoewhas AC, Ayers L, et al. Effects of continuous positive airway pressure therapy withdrawal in patients with obstructive sleep apnea: a randomized controlled trial. Am J Respir Crit Care Med 2011; 184: $1192-1199$

10 Stradling JR, Craig SE, Kohler M, et al. Markers of inflammation: data from the MOSAIC randomised trial of CPAP for minimally symptomatic OSA. Thorax 2015; 70: 181-182.

11 Chirinos JA, Gurubhagavatula I, Teff $\mathrm{K}$, et al. CPAP, weight loss, or both for obstructive sleep apnea. $N$ Engl J Med 2014; 370: 2265-2275.

12 Reid MB, Lannergren J, Westerblad H, et al. Respiratory and limb muscle weakness induced by tumor necrosis factor- $\alpha$ : involvement of muscle myofilaments. Am J Respir Crit Care Med 2002; 166: 479-484.

13 Huxtable AG, Vinit S, Windelborn JA, et al. Systemic inflammation impairs respiratory chemoreflexes and plasticity. Respir Physiol Neurobiol 2011; 178: 482-489.

14 Dunleavy M, Bradford A, O'Halloran $\mathrm{KD}$, et al. Oxidative stress impairs upper airway muscle endurance in an animal model of sleep-disordered breathing. Adv Exp Med Biol 2008; 605: 458-462.

15 Boyd JH, Petrof BJ, Hamid Q, et al. Upper airway muscle inflammation and denervation changes in obstructive sleep apnea. Am J Respir Crit Care Med 2004; 170: 541-546.

16 Berger G, Gilbey P, Hammel I, et al. Histopathology of the uvula and the soft palate in patients with mild, moderate and severe obstructive sleep apnea. Laryngoscope 2002; 112: 357-363.

17 Lindman R, Stahl PS. Abnormal palatopharyngeal muscle morphology in sleep-disordered breathing. J Neurol Sci 2002; 195: 11-23. 